\title{
Aspectos clínicos e epidemiológicos da cefaleia pós-traumática crônica
}

\author{
Rodrigo Antônio Rocha da Cruz Adry ${ }^{1}$, Catarina Couras Lins², \\ Marcio Cesar de Mello Brandão ${ }^{3}$
}

Hospital Geral Roberto Santos e Hospital Geral do Estado, Salvador, BA, Brasil

\section{RESUMO}

Contexto: Existem muitas controvérsias acerca da patogênese da cefaleia pós-traumática, se vem de alterações orgânicas devidas a um trauma cranioencefálico ou se os acometidos dessa condição buscam um ganho secundário. Objetivo: Descrever os aspectos clínicos e epidemiológicos da cefaleia pós-traumática crônica (CPTC) em pacientes atendidos em dois hospitais de referência de Salvador, BA. Métodos: Foram estudados 33 pacientes que preencheram os critérios estabelecidos para CPTC. Resultados: Seis por cento apresentaram frequência de cefaleia inferior a 1 dia por mês; $49 \%$ apresentaram frequência de 1 a 7 dias por mês; $30 \%$, de 8 a 14 dias por mês; 6\%, por mais de 14 dias por mês, e $9 \%$ apresentaram cefaleia diariamente. Quanto à intensidade, $3 \%$ dos pacientes classificaram a cefaleia como leve, $30 \%$ como moderada e $67 \%$ como grave. Vinte e sete por cento caracterizaram sua cefaleia como "em pressão", 61\% como "pulsátil" e 12\% como "em pontada". O diagnóstico da CPTC foi migrânea em $79 \%$ dos pacientes e cefaleia tensional em $21 \%$ dos pacientes. Conclusão: A maior prevalência de migrânea nesses pacientes se deve ao fato de o trabalho ter sido realizado em um dos centros especializados. A uniformidade do padrão manifestado de cefaleia e a média de idade do aparecimento da cefaleia também falam a favor de uma causa orgânica para a CPTC, entretanto faz-se necessária a realização de estudos experimentais que possam comprovar essa teoria analisando os pacientes mais graves para reforçar a hipótese orgânica para origem da CPTC.

\section{PALAVRA-CHAVE}

Cefaleia pós-traumática crônica.

\section{ABSTRACT}

Clinical and epidemiological aspects of chronic post-traumatic headache

Background: Current data describes $75 \%$ to $85 \%$ of chronic post-traumatic headaches (CPTH) have a clinical presentation of tension-type headache. Pathogenesis of post-traumatic headache has been controversial; is it an organic secondary lesion due to head trauma or is simulated on aim of a secondary gain. Objective: To describe the clinical and epidemiological aspects of CPTH in two headache centers in Salvador city (Bahia, Brazil). Method: We studied 33 patients that fulfilled the established criteria for CPTH. Results: The frequency of headache was less than 1 day/month in $6 \%$ of the patients, 1 to 7 days/month in 49\%, 8 to 14 days/month in 30\%, more than 14 days/month in 6\% and $9 \%$ had daily headache. Intensity was analyzed and $3 \%$ of the patients referred mild headache, $30 \%$ moderate and $67 \%$ referred severe headache. Twenty-seven per cent characterized the headache as "pressing", $61 \%$ as "pulsating" and 12\% referred the pain manifesting like "sharp ". Migraine type was diagnosed in $79 \%$ of the patients and tension-type headache in $21 \%$. Conclusion: A high prevalence of migraine may be explained by the recruitment of more severe patients in specialized centers and it supports the hypothesis of an organic pathogenesis for the CPTH. The uniformity of the pattern of headache and the average age of onset of headache also suggest an organic cause for the CPT, however it is necessary to perform experimental studies that can prove this theory.

\section{KEY WORD}

Chronic post-traumatic headache.

1 Serviço de Neurologia e Neurocirurgia do Hospital Geral Roberto Santos e Hospital Geral do Estado, Salvador, BA.

2 Acadêmica de Medicina da Escola Bahiana de Medicina e Saúde Pública, Salvador, BA.

3 Chefe do Serviço de Neurologia e Neurocirurgia do Hospital Geral Roberto Santos e Hospital Geral do Estado, Salvador, BA. 


\section{Introdução}

A cefaleia é o sintoma mais proeminente após uma lesão na cabeça, no pescoço ou no cérebro. A cefaleia pós-traumática (CPT) inicia-se em até sete dias após o traumatismo craniano ou após a recuperação da consciência que se segue ao traumatismo e, se persistir por mais de três meses após a lesão, é denominada cefaleia pós-traumática crônica (CPTC).

A CPTC pode estar associada a outros sintomas como irritabilidade, tontura, insônia, ansiedade, redução da memória, fadiga, redução da concentração, intolerância a ruídos e alterações da personalidade, formando a síndrome pós-traumática (SPT). ${ }^{21-24}$ Todos esses sintomas da SPT contribuem para baixa qualidade de vida e incapacidade nos pacientes. Existem poucas evidências que relacionam a incidência de cefaleia à gravidade do trauma; alguns estudos mostram ser mais frequente em traumatismos cranioencefálicos (TCE) moderados a leves. ${ }^{9}, 13,23,26$ Em estudo retrospectivo realizado na cidade de São Paulo, Maset e col..$^{13}$ encontraram incidência de TCE de 456 internações por cem mil habitantes, com maior acometimento na faixa etária entre 20 e 29 anos. Melo e col..$^{14}$ revisaram 555 prontuários de pacientes admitidos vítimas de TCE em um serviço de emergência na cidade de Salvador em 2001: 7,7\% dos TCEs eram moderados e 63,8\% eram leves, o que traduz grande número de pacientes com potencial risco para desenvolverem a CPTC.

O risco de se desenvolver cefaleia após um TCE varia de $30 \%$ a $90 \%$, de acordo com fatores culturais e com o país estudado. ${ }^{20}$ A CPT torna-se crônica em aproximadamente $60 \%$ dos pacientes, mas geralmente desaparece dentro de 6 a 12 meses após o trauma, persistindo por mais de 1 ano em $33 \%$ dos pacientes e por mais de 3 anos em $15 \%$ a 20\% deles. ${ }^{12}$ Entre as mulheres, a incidência de CPT é duas vezes maior, sendo seu curso de recuperação mais lento e incompleto. ${ }^{1,2,17,19}$

Após um TCE, pode desenvolver-se uma grande variedade de padrões de dor que podem assemelhar-se a cefaleias primárias. A cefaleia do tipo tensional é a mais frequente, ocorrendo em $75 \%$ a $85 \%$ dos pacientes, seguida da migrânea e da cefaleia cervicogênica. ${ }^{24}$

Bigal e col. ${ }^{1}$ estudaram gastos com episódios de cefaleias agudas em unidade de emergência em uma cidade do interior do estado de São Paulo em 1996, chegando ao valor de R \$115,14 por paciente. Em 2001, eles estudaram os gastos do sistema público da mesma cidade com cefaleias e observaram um gasto de $\mathrm{R} \$ 7,59$ por habitante/ano. ${ }^{2}$

A realização desse estudo é justificada pela falta de estudos sobre a CPTC em nosso meio e pelo benefício de se proporcionarem intervenções mais direcionadas favorecendo os pacientes e, no âmbito econômico, o sistema público de saúde. Dessa forma, o objetivo desse estudo foi o de descrever os aspectos clínicos e epidemiológicos da CPTC.

\section{Material e métodos}

Trata-se de um estudo descritivo, em que foi feita uma análise qualitativa e quantitativa, realizada por meio da aplicação de um questionário aos pacientes portadores de CPTC atendidos em dois ambulatórios de cefaleia na cidade de Salvador. O diagnóstico da CPTC baseou-se nos critérios da segunda edição da classificação das cefaleias da Sociedade Internacional de Cefaleia (SIC) de 2005..$^{24}$ Para a aplicação do questionário, foram selecionados os pacientes internados no Hospital Geral Roberto Santos e Hospital Geral do Estado, em Salvador, BA, no período de agosto de 2007 a setembro de 2008.

O questionário foi aplicado a esses pacientes após a assinatura de Termo de Consentimento Livre e Esclarecido pelo paciente.

Por intermédio da aplicação do questionário, foram obtidas informações acerca dos seguintes itens:

1) dados de identificação pessoal;

2) características do trauma - data, tipo, local, tempo decorrido para início da cefaleia, presença de perda de consciência e quanto tempo, exames confirmatórios, presença de contragolpe;

3) características da cefaleia - data do início, topografia das dores, caráter da dor, intensidade das crises, duração das crises, frequência mensal, presença de pródromo, presença de aura, fatores de agravamento/desencadeantes, faltas no trabalho no mês, necessidade de atendimento de emergência, número de comprimidos de resgate usados por mês, mudança nas características da cefaleia, presença de comorbidades, história familiar.

Esse trabalho foi submetido ao Comitê de Ética e Pesquisa da instituição e foi aprovado para realização.

\section{Resultados}

Dos 118 pacientes portadores de cefaleia atendidos no período de coleta, 33 (28\%) completaram os critérios para CPTC. Destes, a média de idade foi de 42 anos, variando de 13 a 70 anos, sendo $61 \%$ do sexo feminino. 
O tempo de história da CPTC variou de seis meses a cinco anos; radiografia do crânio havia sido feita em oito pacientes (24\%) e, em quatro (12\%), tomografia computadorizada (TC) de crânio. Todos os exames de imagem estavam normais.

Dois pacientes $(6 \%)$ apresentaram frequência da cefaleia menor que um dia por mês, 16 pacientes (49\%) apresentaram frequência de um dia a sete dias por mês, dez pacientes (30\%), de 8 a 14 dias por mês, dois pacientes $(6 \%)$, por mais de 14 dias por mês, e três pacientes (9\%) apresentaram cefaleia diária (Tabela 1).

\begin{tabular}{lcc}
\hline \multicolumn{2}{c}{ Tabela 1 } & \\
& Frequência mensal da & CPTC \\
\hline & N & $\%$ \\
Menos que 1 dia por mês & 2 & 6 \\
1-7 dias por mês & 16 & 49 \\
8-14 dias por mês & 10 & 30 \\
Mais de 14 dias por mês & 2 & 6 \\
Contínua & 3 & 9 \\
Total & 33 & 100 \\
\hline
\end{tabular}

Em relação à intensidade da cefaleia, um paciente (3\%) a classificou como leve, dez pacientes (30\%), como moderada, e 22 pacientes $(67 \%)$, como grave. Nove pacientes $(27 \%)$ caracterizaram sua cefaleia como "em pressão", 20 pacientes $(61 \%)$ a caracterizaram como "pulsátil", e 4 pacientes (12\%), como "em pontada".

Os principais sintomas associados à cefaleia foram ansiedade, cintilações, depressão, dor cervical, escotomas, fonofobia, fotofobia, irritabilidade, mal-estar, náusea, palpitação, parestesias, sudorese, tontura e vômito. Estudando-se as características das cefaleias de acordo com a classificação da SIC de 2005, foram encontrados os seguintes diagnósticos nos pacientes estudados: migrânea com aura em $12 \%$ dos pacientes, migrânea sem aura em $67 \%$ dos pacientes e cefaleia tensional em $21 \%$ dos pacientes.

\section{Discussão}

A etiologia da CPTC não é conhecida. Há controvérsia quanto a sua origem orgânica ou psicológica. Estudos anatomopatológicos post mortem do tecido cerebral encontraram lesões microscópicas incluindo a lesão axonal difusa. ${ }^{6,16}$ Estudos laboratoriais mostram alterações após TCE, sugerindo a presença de marcadores de lesão cerebral. ${ }^{10}$ Estudos de imagens de ressonância magnética encontraram lesões parenquimatosas, ${ }^{11}$ e outros utilizando a tomografia computadorizada com emissão de pósitrons revelaram fluxo sanguíneo cerebral diminuído após o trauma. Outros trabalhos mostraram fluxo sanguíneo cerebral regional anormal e assimétrico e zonas focais de perfusão diminuída três anos após a lesão em pacientes com CPTC. ${ }^{7,18}$ Alterações metabólicas foram descritas, ${ }^{5,22}$ entretanto, juntamente a trabalhos de eletroencefalograma, de potenciais evocados e estudo de líquido cefalorraquidiano, se mostraram inconclusivas.

Por outro lado, a depressão e o estresse parecem influenciar a CPTC. ${ }^{8}$ Estudos recentes revelam que a maioria das pessoas vítimas de um TCE moderado que interromperam a busca por compensação financeira em razão de dor e sofrimento apresentaram queda da incidência de CPTC e melhor prognóstico. ${ }^{3}$ Em países onde não existem leis que regulam a compensação financeira por trauma, a incidência de CPTC é menor. ${ }^{15}$

Este estudo foi realizado em dois centros especializados em acompanhamento de cefaleias, o que possibilitou estudo da CPTC em uma população mais variada, entretanto, houve um recrutamento não intencional dos casos mais graves, isto é, aqueles que recorrem a essas instituições.

A taxa da CPTC neste estudo foi de $28 \%$ do total de pacientes com cefaleia dos ambulatórios, o que coloca o TCE como uma importante e frequente causa de cefaleia crônica.

A CPTC parece predominar no sexo feminino, ${ }^{17,24}$ geralmente na média de 2:1. Em nosso estudo $60 \%$ dos pacientes com CPTC eram mulheres.

A ausência de alterações nos exames de imagem foi um achado desse estudo, sugerindo um TCE leve. Alguns estudos relatam uma relação inversa entre a incidência da CPT e a gravidade do TCE., $93,23,26$

A migrânea foi o padrão primário mais frequente de CPTC, correspondendo a 76\% das CPTCs contra $24 \%$ de cefaleia tensional. Esse dado está em desacordo com os dados mundiais que remetem $75 \%$ a $80 \%$ das CPTCs ao padrão tensional. ${ }^{1,23}$ Uma frequência maior de migrânea não sugere uma etiologia psicogênica para a CPTC, pois antes do trauma os pacientes nunca haviam relatado uma cefaleia tão rica em detalhes como a migrânea. Segundo Weiss e col., ${ }^{25}$ o trauma não parece atuar como "gatilho" bioquímico para o aparecimento da CPT, pois em sua série de casos os TCEs eram leves, mas a hipótese é de que seja um fator de risco juntamente a predisposição genética, idade, gênero, estresse. Em 2007, Couch e col. ${ }^{4}$ mostraram que história de TCE aumenta em aproximadamente $15 \%$ os casos de cefaleia crônica diária (CCD) em um estudo de base populacional. O risco durante a vida de CCD aumenta com o aumento do número de TCE.

A média de idade da população estudada foi mais alta do que a população com migrânea idiopática, dado 
semelhante ao encontrado por Weiss e col., ${ }^{25}$ reforçando a hipótese orgânica da CPTC.

Um total de $67 \%$ dos pacientes classificou sua cefaleia como grave, compatível com o padrão de dor da migrânea.

\section{Conclusão}

A uniformidade do padrão de cefaleia manifestado e a média de idade do aparecimento da cefaleia falam a favor de uma causa orgânica para a CPTC, entretanto faz-se necessária a realização de estudos experimentais que possam comprovar essa teoria.

\section{Referências}

1. Bigal ME, Fernandes LC, Bordini CA, Speciali JG. Custos hospitalares das cefaléias agudas em uma unidade de emergência pública brasileira. Arq Neuropsiquiatr. 2000;58:664-70.

2. Bigal ME, Fernandes LC, Bordini CA, Speciali JG.. Prevalence and costs of headaches for the public health system in a town in the interior of the state of São Paulo. Arq Neuropsiquiatr. 2001;59:504-11.

3. Cassidy JD, Carroll LJ, Cote P, Lemstra M, Berglund A, Nygren A. Effect of eliminating of compensation for pain and suffering on the outcome of insurance claims for whiplash injury. N Engl J Med. 2000;342:1179-86.

4. Couch JR, Lipton RB, Stewart WF, Scher Al. Head or neck injury increases the risk of chronic daily headache: A population-based study. Neurology. 2007;69:1169-77.

5. Garnett MR. Early proton magnetic resonance spectroscopy in normal-appearing brain correlates with injury severity in patients following traumatic brain injury: a magnetic resonance spectroscopy study. Brain. 2000;123:1043-9.

6. Gennarelli TA, Thibault LE, Adams JH, Graham DI, Thompson CJ, Marcincin RP. Diffuse axonal injury and traumatic coma in the primate. Ann Neurol. 1982;12:564-74.

7. Gilkey SJ, Ramadan NM, Aurora TK, Welch KM. Cerebral blood flow is abnormal in chronic posttraumatic headache. Headache. 1997;37:583-7.

8. Ham LP, Andrasik F, Packard RC, Bundrick CM. Psychopathology in individuals with posttraumatic headache and other pain types. Cephalalgia. 1994;14:118-26.

9. Kay DW, Kerr TA, Lassman LP. Brain trauma and the postconcussional syndrome. Lancet. 1971;2:1052-5.

10. Kruijk JR. Prediction of post-traumatic complains after mild traumatic brain injury: early symptoms and biochemical markers. J Neurol Neurosurg Psychiatry. 2002;73:727-32.
11. Levin HS, Amparo E, Eisenberg HM, Miner ME, High WM Jr, Ewing-Cobbs L, et al. Magnetic resonance imaging and computadorized tomography in relation to the neurobehavioral sequelae of mild and moderate head injuries. J Neurosurg. 1987;66:706-13.

12. Marcus DA. Disability and chronic posttraumatic headache. Headache. 2003;43:117-21.

13. Maset, Angelo; Andrade, Almir; Martucci, Salvador C; Frederico, Luis Mauro Epidemiologic features of head injury in Brazil. Arq Bras Neurocir 1993;12:293-302.

14. Melo JRT, Silva RA, Moreira JR, Duarte E. Característica dos pacientes com trauma cranioencefálico na cidade de Salvador, Bahia, Brasil. Arq Neuropsiquiatr. 2004;62:711-5.

15. Obelieniene D, Schrader H, Bovim G, Miseviciene I, Sand T. Pain after whiplash: A prospective controlled inception cohort study. J Neurol Neurosurg Psychiatry. 1999;66:279-83.

16. Oppenheimer DR. Microscopic lesions in the brain following head injury. J Neurol Neurosurg Psychiatry. 1969;31:299306.

17. Pascual-Lozano AM, Salvador-Aliaga A, Láinez-Andrés JM La cefalea postraumática: Fisiopatología, aspectos clínicos, diagnósticos y terapêuticos. Neurologia. 2005;20:133-42.

18. Ramadan NM, Noris LL, Schultz RL. Abnormal cerebral blood flow correlates with disability to chronic posttraumatic headache. J Neuroimaging. 1995;5:68.

19. Sheftell FD. Post-traumatic headache: emphasis on chronic types following mild closed head injury. Neurol Sci. 2007;28:203-7.

20. Solomon S. Posttraumatic headache. Med Clin North Am. 2001;85:987-96.

21 Solomon S. Chronic post-traumatic neck and head pain. Headache 2005;45:53-7.

22. Son BC. Metabolic changes in periconcusional edematous areas in mild head injury evaluated by $1 \mathrm{H}-\mathrm{MRS}$. Acta Neurochir (Suppl). 2000;76:13-6.

23. Souza JA, Moreira Filho PF, Jevoux CC. Cefaléia póstraumática crônica em traumatismos crânio-encefálicos leves. Arq Neuropsiquiatr. 1999;57:243-8.

24. Subcomitê de Classificação das Cefaléias da Sociedade Internacional de Cefaléia. Classificação internacional das cefaléias. 2a. Ed. Traduzido pela Sociedade Brasileira de Cefaléia. São Paulo: Alaúie Editorial Ltda. 2006. p.106-17.

25. Weiss HD, Stern BJ, Goldberg J. Post-traumatic migraine: chronic migraine precipitated by minor head or neck trauma. Headache. 1991;31:451-6.

26. Yamaguchi M. Incidence of headache and severity of head injury. Headache. 1992;32:427-31.

Original recebido em setembro de 2009

Aceito para publicação em janeiro de 2010

Endereço para correspondência

Rodrigo Antônio Rocha da Cruz Adry

Av. Sete de Setembro, 690, ap. 406

40060-001 - Salvador, Bahia

E-mail: rodrigoadry@hotmail.com. 